\title{
High Accurate Fourth-Order Finite Difference Solutions of the Three Dimensional Poisson's Equation in Cylindrical Coordinate
}

\author{
Alemayehu Shiferaw, Ramesh Chand Mittal \\ Department of Mathematics, Indian Institute of Technology, Roorkee, India \\ Email: abelhaim@gmail.com, mittalrc@gmail.com
}

Received 5 November 2013; revised 5 December 2013; accepted 16 December 2013

Copyright (C) 2014 by authors and Scientific Research Publishing Inc.

This work is licensed under the Creative Commons Attribution International License (CC BY).

http://creativecommons.org/licenses/by/4.0/

(c) (i) Open Access

\begin{abstract}
In this work, by extending the method of Hockney into three dimensions, the Poisson's equation in cylindrical coordinates system with the Dirichlet's boundary conditions in a portion of a cylinder for $r \neq 0$ is solved directly. The Poisson equation is approximated by fourth-order finite differences and the resulting large algebraic system of linear equations is treated systematically in order to get a block tri-diagonal system. The accuracy of this method is tested for some Poisson's equations with known analytical solutions and the numerical results obtained show that the method produces accurate results.
\end{abstract}

\section{Keywords}

Poisson's Equation; Tri-Diagonal Matrix; Fourth-Order Finite Difference Approximation; Hockney's Method; Thomas Algorithm

\section{Introduction}

The three-dimensional Poisson's equation in cylindrical coordinates $(r, \theta, z)$ is given by

$$
U_{r r}+\frac{1}{r} U_{r}+\frac{1}{r^{2}} U_{\theta \theta}+U_{z z}=f(r, \theta, z)
$$

has a wide range of application in engineering and science fields (especially in physics).

How to cite this paper: Shiferaw, A. and Mittal, R.C. (2014) High Accurate Fourth-Order Finite Difference Solutions of the Three Dimensional Poisson's Equation in Cylindrical Coordinate. American Journal of Computational Mathematics, 4, 73-86. http://dx.doi.org/10.4236/jasmi.2014.42007 
In physical problems that involve a cylindrical surface (for example, the problem of evaluating the temperature in a cylindrical rod), it will be convenient to make use of cylindrical coordinates. For the numerical solution of the three dimensional Poisson's equation in cylindrical coordinates system, several attempts have been made in particular for physical problems that are related directly or indirectly to this equation. For instance, Lai [1] developed a simple compact fourth-order Poisson solver on polar geometry based on the truncated Fourier series expansion, where the differential equations of the Fourier coefficients are solved by the compact fourth-order finite difference scheme; Mittal and Gahlaut [2] have developed high order finite difference schemes of second- and fourth- order in polar coordinates using a direct method similar to Hockney's method; Mittal and Gahlaut [3] developed a second- and fourth-order finite difference scheme to solve Poisson's equation in the case of cylindrical symmetry; Alemayehu and Mittal [4] have derived a second-order finite difference approximation scheme to solve the three dimensional Poisson's equation in cylindrical coordinates by extending Hockney's method; Tan [5] developed a spectrally accurate solution for the three dimensional Poisson's equation and Helmholtz's equation using Chebyshev series and Fourier series for a simple domain in a cylindrical coordinate system; Iyengar and Manohar [6] derived fourth-order difference schemes for the solution of the Poisson equation which occurs in problems of heat transfer; Iyengar and Goyal [7] developed a multigrid method in cylindrical coordinates system; Lai and Tseng [8] have developed a fourth-order compact scheme, and their scheme relies on the truncated Fourier series expansion, where the partial differential equations of Fourier coefficients are solved by a formally fourth-order accurate compact difference discretization. The need to obtain the best solution for the three dimensional Poisson's equation in cylindrical coordinates system is still in progress.

In this paper, we develop a fourth-order finite difference approximation scheme and solve the resulting large algebraic system of linear equations systematically using block tridiagonal system [9] [10] and extend the Hockney's method [9] [11] to solve the three dimensional Poisson's equation on Cylindrical coordinates system.

\section{Finite Difference Approximation}

Consider the three dimensional Poisson's equation in cylindrical coordinates $(r, \theta, z)$ given by

$$
\frac{\partial^{2} U}{\partial r^{2}}+\frac{1}{r} \frac{\partial U}{\partial r}+\frac{1}{r^{2}} \frac{\partial^{2} U}{\partial \theta^{2}}+\frac{\partial^{2} U}{\partial z^{2}}=f(r, \theta, z) \text { on } D
$$

and the boundary condition

$$
U(r, \theta, z)=g(r, \theta, z) \text { on } C
$$

where $C$ is the boundary of $D$ and $D$ is

$D_{1}=$

$\left\{(r, \theta, z): R_{0}<r<R_{1}, a<z<b, \theta_{0}<\theta<\theta_{1}, \theta_{0}<\theta_{1}<2 \pi\right\}$ and $D_{2}=\left\{(r, \theta, z): R_{0}<r<R_{1}, a<z<b, 0 \leq \theta<2 \pi\right\}$

Consider Figure 1 as the geometry of the problem. Let $u(r, \theta, z)$ be discretized at the point $\left(r_{i}, \theta_{j}, z_{k}\right)$ and for simplicity write a point $\left(r_{i}, \theta_{j}, z_{k}\right)$ as $(i, j, k)$ and $u\left(r_{i}, \theta_{j}, z_{k}\right)$ as $u_{i, j, k}$.

Assume that there are $M$ points in the direction of $r, N$ points in $\theta$ and $P$ points in the $z$ directions to form the mesh, and let the step size along the direction of $r$ be $\Delta r$, of $\theta$ be $\Delta \theta$ and $z$ be $\Delta z$.

Here $r_{i}=R_{0}+i \Delta r, \theta_{j}=\theta_{0}+j \Delta \theta$ and $z_{k}=a+k \Delta z$

Where $i=1,2, \cdots, M, j=1,2, \cdots, N$ and $k=1,2, \cdots, P$.

When $r=0$ is an interior or a boundary point of (2), then the Poisson's equation becomes singular and to take care of the singularity a different approach will be taken. Thus in this paper we consider only for the case $r \neq 0$.

Using the approximations that

$$
\begin{aligned}
& \left(\frac{\partial^{2} U}{\partial r^{2}}\right)_{i, j, k}=\frac{1}{(\Delta r)^{2}}\left(1+\frac{1}{12} \delta_{r}^{2}\right)^{-1} \delta_{r}^{2} U_{i, j, k}+O\left((\Delta r)^{4}\right) \\
& \left(\frac{\partial^{2} U}{\partial \theta^{2}}\right)_{i, j, k}=\frac{1}{(\Delta \theta)^{2}}\left(1+\frac{1}{12} \delta_{\theta}^{2}\right)^{-1} \delta_{\theta}^{2} U_{i, j, k}+O\left((\Delta \theta)^{4}\right)
\end{aligned}
$$




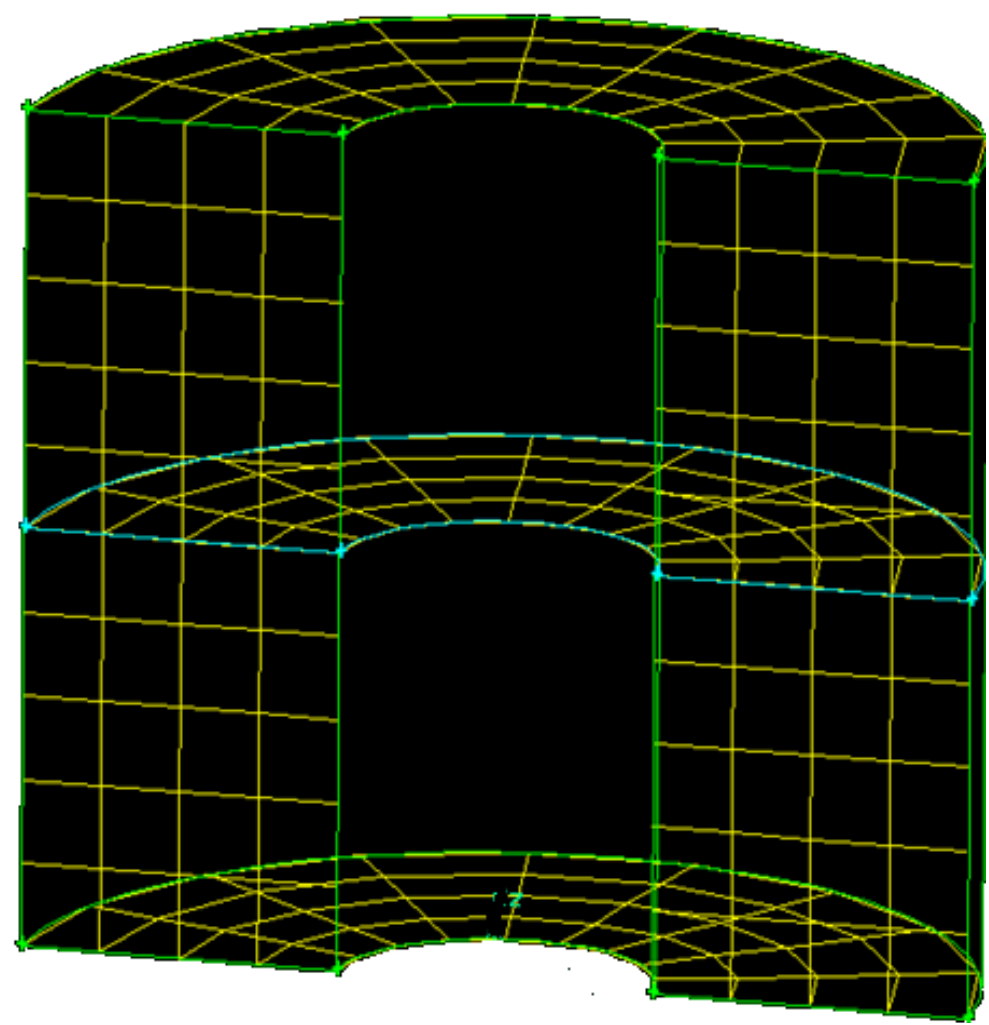

Figure 1. Portion of a cylinder.

$$
\left(\frac{\partial^{2} U}{\partial z^{2}}\right)_{i, j, k}=\frac{1}{(\Delta z)^{2}}\left(1+\frac{1}{12} \delta_{z}^{2}\right)^{-1} \delta_{z}^{2} U_{i, j, k}+O\left((\Delta z)^{4}\right)
$$

Now using (3), (4) and (5), we get (Refer the work of Mittal and Ghalaut in [2])

From (1) consider only the approximation of the sum of the first and the third terms, that is, the sum of $\frac{\partial^{2} U}{\partial r^{2}}$ and $\frac{1}{r_{i}^{2}} \frac{\partial^{2} U}{\partial \theta^{2}}$

$$
\begin{aligned}
& \left(\frac{\partial^{2} U}{\partial r^{2}}+\frac{1}{r_{i}^{2}} \frac{\partial^{2} U}{\partial \theta^{2}}\right)_{i, j, k} \\
= & \frac{1}{12(\Delta r)^{2}}\left[-20\left(1+\frac{\omega}{r_{i}^{2}}\right) U_{i, j, k}+2\left(5-\frac{\omega}{r_{i}^{2}}\right)\left(U_{i+1, j, k}+U_{i-1, j, k}\right)+2\left(\frac{5 \omega}{r_{i}^{2}}-1\right)\left(U_{i, j+1, k}+U_{i, j-1, k}\right)\right. \\
& \left.+\left(1+\frac{\omega}{r_{i}^{2}}\right)\left(U_{i+1, j+1, k}+U_{i+1, j-1, k}+U_{i-1, j+1, k}+U_{i-1, j-1, k}\right)\right]-\frac{1}{12}\left(\frac{\partial^{2}}{\partial r^{2}}+\frac{1}{r_{i}^{2}} \frac{\partial^{2}}{\partial \theta^{2}}\right) \\
& \left((\Delta r)^{2} \frac{\partial^{2}}{\partial r^{2}}+(\Delta \theta)^{2} \frac{\partial^{2}}{\partial \theta^{2}}\right) U_{i, j, k}+O\left((\Delta r)^{4}+(\Delta \theta)^{4}\right)
\end{aligned}
$$

where $\omega=\frac{(\Delta r)^{2}}{(\Delta \theta)^{2}}$

Again from (1) consider only the approximation of the sum of the first and the fourth terms, that is, the sum of $\frac{\partial^{2} U}{\partial r^{2}}$ and $\frac{\partial^{2} U}{\partial z^{2}}$, and we get 


$$
\begin{aligned}
& \left(\frac{\partial^{2} U}{\partial r^{2}}+\frac{\partial^{2} U}{\partial z^{2}}\right)_{i, j, k} \\
& =\frac{1}{12(\Delta r)^{2}}\left[\left(1+\frac{(\Delta r)^{2}}{(\Delta z)^{2}}\right)\left(U_{i+1, j, k+1}+U_{i+1, j, k-1}+U_{i-1, j, k+1}+U_{i-1, j, k-1}\right)\right. \\
& \left.\quad+2\left(5-\frac{(\Delta r)^{2}}{(\Delta z)^{2}}\right)\left(U_{i+1, j, k}+U_{i-1, j, k}\right)+2\left(5 \frac{(\Delta r)^{2}}{(\Delta z)^{2}}-1\right)\left(U_{i, j+1, k}+U_{i, j-1, k}\right)-20\left(1+\frac{(\Delta r)^{2}}{(\Delta z)^{2}}\right) U_{i, j, k}\right] \\
& -\frac{1}{12}\left(\frac{\partial^{2}}{\partial r^{2}}+\frac{\partial^{2}}{\partial z^{2}}\right)\left((\Delta r)^{2} \frac{\partial^{2}}{\partial r^{2}}+(\Delta z)^{2} \frac{\partial^{2}}{\partial z^{2}}\right) U_{i, j, k}+O\left((\Delta r)^{4}+(\Delta z)^{4}\right)
\end{aligned}
$$

Once again from (1) consider only the approximation of the sum of the second and the fourth terms, that is, the sum of $\frac{1}{r_{i}^{2}} \frac{\partial^{2} U}{\partial \theta^{2}}$ and $\frac{\partial^{2} U}{\partial z^{2}}$; to get

$$
\begin{aligned}
& \left(\frac{1}{r_{i}^{2}} \frac{\partial^{2} U}{\partial \theta^{2}}+\frac{\partial^{2} U}{\partial z^{2}}\right)_{i, j, k} \\
& =\frac{1}{12}\left[\left(\frac{1}{\left(r_{i} \Delta \theta\right)^{2}}+\frac{1}{(\Delta z)^{2}}\right)\left(U_{i, j+1, k+1}+U_{i, j+1, k-1}+U_{i, j-1, k+1}+U_{i, j-1, k-1}\right)+2\left(\frac{5}{\left(r_{i} \Delta \theta\right)^{2}}-\frac{1}{(\Delta z)^{2}}\right)\left(U_{i, j+1, k}+U_{i, j-1, k}\right)\right. \\
& \left.+2\left(\frac{5}{(\Delta z)^{2}}-\frac{1}{\left(r_{i} \Delta \theta\right)^{2}}\right)\left(U_{i, j, k+1}+U_{i, j, k-1}\right)-20\left(\frac{1}{\left(r_{i} \Delta \theta\right)^{2}}+\frac{1}{(\Delta z)^{2}}\right) U_{i, j, k}\right] \\
& -\frac{1}{12}\left(\frac{1}{r_{i}^{2}} \frac{\partial^{2}}{\partial \theta^{2}}+\frac{\partial^{2}}{\partial z^{2}}\right)\left((\Delta \theta)^{2} \frac{\partial^{2}}{\partial r^{2}}+(\Delta z)^{2} \frac{\partial^{2}}{\partial z^{2}}\right) U_{i, j, k}+O\left((\Delta \theta)^{4}+(\Delta z)^{4}\right)
\end{aligned}
$$

Again taking the approximation of the term $\frac{\partial U}{\partial r}$ by

$$
\begin{aligned}
\left(\frac{\partial U}{\partial r}\right)_{i, j, k}= & \frac{\phi \delta_{2 r}\left(U_{i, j+1, k}+U_{i, j-1, k}+U_{i, j, k+1}+U_{i, j, k-1}\right)+(1-4 \phi) \delta_{2 r} U_{i, j, k}}{2 \Delta r} \\
& -\frac{1}{3}(\Delta r)^{2} \frac{\partial^{3} U_{i, j, k}}{\partial r^{3}}-\phi(\Delta \theta)^{2} \frac{\partial^{3} U_{i, j, k}}{\partial r \partial \theta^{2}}-\phi(\Delta z)^{2} \frac{\partial^{3} U_{i, j, k}}{\partial r \partial z^{2}} \\
& +O\left((\Delta r)^{4}+(\Delta \theta)^{4}+(\Delta z)^{4}\right), 0 \leq \phi \leq 1
\end{aligned}
$$

Equation (9) implying that

$$
\begin{aligned}
\frac{1}{r_{i}}\left(\frac{\partial U}{\partial r}\right)_{i, j, k}= & \frac{\phi \delta_{2 r}\left(U_{i, j+1, k}+U_{i, j-1, k}+U_{i, j, k+1}+U_{i, j, k-1}\right)+(1-4 \phi) \delta_{2 r} U_{i, j, k}}{2 r_{i} \Delta r} \\
& -\frac{1}{3 r_{i}}(\Delta r)^{2} \frac{\partial^{3} U_{i, j, k}}{\partial r^{3}}-\phi(\Delta \theta)^{2} \frac{1}{r_{i}} \frac{\partial^{3} U_{i, j, k}}{\partial r \partial \theta^{2}} \\
& -\phi(\Delta z)^{2} \frac{1}{r_{i}} \frac{\partial^{3} U_{i, j, k}}{\partial r \partial z^{2}}+O\left((\Delta r)^{4}+(\Delta \theta)^{4}+(\Delta z)^{4}\right)
\end{aligned}
$$

Now letting $\alpha=\frac{(\Delta r)^{2}}{(\Delta z)^{2}}$ and adding (6), (7), (8) and twice of (10), we get 


$$
\begin{aligned}
2\left(\frac{\partial^{2} U}{\partial r^{2}}+\frac{1}{r_{i}} \frac{\partial U}{\partial r}+\frac{1}{r_{i}^{2}} \frac{\partial^{2} U}{\partial \theta^{2}}+\frac{\partial^{2} U}{\partial z^{2}}\right)_{i, j, k} \\
=\frac{1}{12(\Delta r)^{2}}\left[\left(1+\frac{\omega}{r_{i}^{2}}\right)\left(U_{i+1, j+1, k}+U_{i+1, j-1, k}+U_{i-1, j+1, k}+U_{i-1, j-1, k}\right)+2\left(5-\frac{\omega}{r_{i}^{2}}\right)\left(U_{i+1, j, k}+U_{i-1, j, k}\right)\right. \\
+2\left(\frac{5 \omega}{r_{i}^{2}}-1\right)\left(U_{i, j+1, k}+U_{i, j-1, k}\right)+(1+\alpha)\left(U_{i+1, j, k+1}+U_{i+1, j, k-1}+U_{i-1, j, k+1}+U_{i-1, j, k-1}\right) \\
\left.+2(5-\alpha)\left(U_{i+1, j, k}+U_{i-1, j, k}\right)+2(5 \alpha-1)\left(U_{i, j+1, k}+U_{i, j-1, k}\right)-20\left(2+\alpha+\frac{\omega}{r_{i}^{2}}\right) U_{i, j, k}\right] \\
+\frac{1}{12}\left[\left(\frac{1}{\left(r_{i} \Delta \theta\right)^{2}}+\frac{1}{(\Delta z)^{2}}\right)\left(U_{i, j+1, k+1}+U_{i, j+1, k-1}+U_{i, j-1, k+1}+U_{i, j-1, k-1}\right)-20\left(\frac{1}{\left(r_{i} \Delta \theta\right)^{2}}+\frac{1}{(\Delta z)^{2}}\right) U_{i, j, k}\right. \\
+2\left(\frac{5}{\left(r_{i} \Delta \theta\right)^{2}}-\frac{1}{(\Delta z)^{2}}\right)\left(U_{i, j+1, k}+U_{i, j-1, k}\right)+2\left(\frac{5}{(\Delta z)^{2}}-\frac{1}{\left(r_{i} \Delta \theta\right)^{2}}\right)\left(U_{i, j, k+1}+U_{i, j, k-1}\right) \\
+\frac{1}{12}\left(\frac{\partial^{2}}{\partial r^{2}}+\frac{1}{r_{i}^{2}} \frac{\partial^{2}}{\partial \theta^{2}}\right)\left((\Delta r)^{2} \frac{\partial^{2}}{\partial r^{2}}+(\Delta \theta)^{2} \frac{\partial^{2}}{\partial \theta^{2}}\right) U_{i, j, k}-\frac{1}{12}\left(\frac{\partial^{2}}{\partial r^{2}}+\frac{\partial^{2}}{\partial z^{2}}\right)\left((\Delta r)^{2} \frac{\partial^{2}}{\partial r^{2}}+(\Delta z)^{2} \frac{\partial^{2}}{\partial z^{2}}\right) U_{i, j, k} \\
+\frac{1}{12}\left(\frac{1}{r_{i}^{2}} \frac{\partial^{2}}{\partial \theta^{2}}+\frac{\partial^{2}}{\partial z^{2}}\right)\left((\Delta \theta)^{2} \frac{\partial^{2}}{\partial r^{2}}+(\Delta z)^{2} \frac{\partial^{2}}{\partial z^{2}}\right) U_{i, j, k}+\frac{\phi \delta_{2 r}\left(U_{i, j+1, k}+U_{i, j-1, k}+U_{i, j, k+1}+U_{i, j, k-1}\right)}{\Delta r} \\
+\frac{(1-4 \phi) \delta_{2 r} U_{i, j, k}}{\Delta r}-\frac{1}{3}(\Delta r)^{2} \frac{\partial^{3} U_{i, j, k}}{\partial r^{3}}-\phi(\Delta \theta)^{2} \frac{\partial^{3} U_{i, j, k}}{\partial r \partial \theta^{2}}-\phi(\Delta z)^{2} \frac{\partial^{3} U_{i, j, k}}{\partial r \partial z^{2}}+O\left((\Delta r)^{4}+(\Delta \theta)^{4}+(\Delta z)^{4}\right)
\end{aligned}
$$

Now choose $\phi=\frac{1}{12}$ and consider the following terms in (11)

$$
\begin{aligned}
- & \frac{1}{12}\left(\frac{\partial^{2}}{\partial r^{2}}+\frac{1}{r_{i}^{2}} \frac{\partial^{2}}{\partial \theta^{2}}\right)\left((\Delta r)^{2} \frac{\partial^{2}}{\partial r^{2}}+(\Delta \theta)^{2} \frac{\partial^{2}}{\partial \theta^{2}}\right) U_{i, j, k} \\
- & \frac{1}{12}\left(\frac{\partial^{2}}{\partial r^{2}}+\frac{\partial^{2}}{\partial z^{2}}\right)\left((\Delta r)^{2} \frac{\partial^{2}}{\partial r^{2}}+(\Delta z)^{2} \frac{\partial^{2}}{\partial z^{2}}\right) U_{i, j, k}-\frac{1}{3 r_{i}}(\Delta r)^{2} \frac{\partial^{3} U_{i, j, k}}{\partial r^{3}} \\
- & \frac{1}{12}\left(\frac{1}{r_{i}^{2}} \frac{\partial^{2}}{\partial \theta^{2}}+\frac{\partial^{2}}{\partial z^{2}}\right)\left((\Delta \theta)^{2} \frac{\partial^{2}}{\partial r^{2}}+(\Delta z)^{2} \frac{\partial^{2}}{\partial z^{2}}\right) U_{i, j, k}-\frac{1}{12 r_{i}}\left((\Delta \theta)^{2} \frac{\partial^{3} U_{i, j, k}}{\partial r \partial \theta^{2}}+(\Delta z)^{2} \frac{\partial^{3} U_{i, j, k}}{\partial r \partial z^{2}}\right) \\
= & -\frac{1}{3 r_{i}}(\Delta r)^{2} \frac{\partial^{3} U}{\partial r^{3}}-\frac{1}{12}\left((\Delta r)^{2} \frac{\partial^{2}}{\partial r^{2}}+(\Delta \theta)^{2} \frac{\partial^{2}}{\partial \theta^{2}}+(\Delta z)^{2} \frac{\partial^{2}}{\partial z^{2}}\right)\left(\frac{\partial^{2}}{\partial r^{2}}+\frac{1}{r_{i}^{2}} \frac{\partial^{2}}{\partial \theta^{2}}+\frac{\partial^{2}}{\partial z^{2}}\right) U_{i, j, k} \\
& -\frac{1}{12}\left((\Delta r)^{2} \frac{\partial^{2}}{\partial r^{2}}\left(\frac{\partial^{2}}{\partial r^{2}}\right)+(\Delta \theta)^{2} \frac{\partial^{2}}{\partial \theta^{2}}\left(\frac{\partial^{2}}{\partial \theta^{2}}\right)+(\Delta z)^{2} \frac{\partial^{2}}{\partial z^{2}}\left(\frac{\partial^{2}}{\partial z^{2}}\right)\right) U_{i, j, k} \\
& -\frac{1}{12 r_{i}}\left((\Delta \theta)^{2} \frac{\partial^{3} U_{i, j, k}}{\partial r \partial \theta^{2}}+(\Delta z)^{2} \frac{\partial^{3} U_{i, j, k}}{\partial r \partial z^{2}}\right) \\
= & -\frac{1}{12}\left((\Delta r)^{2} \frac{\partial^{2}}{\partial r^{2}}+(\Delta \theta)^{2} \frac{\partial^{2}}{\partial \theta^{2}}+(\Delta z)^{2} \frac{\partial^{2}}{\partial z^{2}}\right)\left(\frac{\partial^{2}}{\partial r^{2}}+\frac{1}{r_{i}^{2}} \frac{\partial^{2}}{\partial \theta^{2}}+\frac{\partial^{2}}{\partial z^{2}}\right) U_{i, j, k} \\
& -\frac{1}{12}\left(\frac{1}{r_{i}} \frac{\partial}{\partial r}\left((\Delta r)^{2} \frac{\partial^{2}}{\partial r^{2}}+(\Delta \theta)^{2} \frac{\partial^{2}}{\partial \theta^{2}}+(\Delta z)^{2} \frac{\partial^{2}}{\partial z^{2}}\right) U_{i, j, k}\right)-\frac{1}{4 r_{i}}(\Delta r)^{2} \frac{\partial^{3} U_{i, j, k}}{\partial r^{3}} \\
= & -\frac{1}{12}\left((\Delta r)^{2} \frac{\partial^{2}}{\partial r^{2}}+(\Delta \theta)^{2} \frac{\partial^{2}}{\partial \theta^{2}}+(\Delta z)^{2} \frac{\partial^{2}}{\partial z^{2}}\right)\left(\frac{\partial^{2}}{\partial r^{2}}+\frac{1}{r_{i}} \frac{\partial}{\partial r}+\frac{1}{r_{i}^{2}} \frac{\partial^{2}}{\partial \theta^{2}}+\frac{\partial^{2}}{\partial z^{2}}\right) U_{i, j, k}-\frac{1}{4 r_{i}}(\Delta r)^{2} \frac{\partial^{3} U_{i, j, k}}{\partial r^{3}}
\end{aligned}
$$


Again we can write the term $-\frac{1}{4 r_{i}}(\Delta r)^{2} \frac{\partial^{3} U_{i, j, k}}{\partial r^{3}}$ in (12) as

$$
\begin{aligned}
- & \frac{(\Delta r)^{2}}{4 r_{i}} \frac{\partial^{3} U_{i, j, k}}{\partial r^{3}} \\
= & -\frac{(\Delta r)^{2}}{4 r_{i}} \frac{\partial}{\partial r}\left(\frac{\partial^{2}}{\partial r^{2}}+\frac{1}{r_{i}} \frac{\partial}{\partial r}+\frac{1}{r_{i}^{2}} \frac{\partial^{2}}{\partial \theta^{2}}+\frac{\partial^{2}}{\partial z^{2}}\right) U_{i, j, k} \\
& +\frac{(\Delta r)^{2}}{4 r_{i}} \frac{\partial}{\partial r}\left(\frac{1}{r_{i}} \frac{\partial U_{i, j, k}}{\partial r}\right)+\frac{(\Delta r)^{2}}{4 r_{i}} \frac{\partial}{\partial r}\left(\frac{1}{r_{i}^{2}} \frac{\partial^{2} U_{i, j, k}}{\partial \theta^{2}}\right)+\frac{(\Delta r)^{2}}{4 r_{i}} \frac{\partial}{\partial r}\left(\frac{\partial^{2} U_{i, j, k}}{\partial z^{2}}\right) \\
= & -\frac{(\Delta r)^{2}}{4 r_{i}} \frac{\partial f}{\partial r}-\frac{(\Delta r)^{2}}{4 r_{i}^{3}} \frac{\partial U_{i, j, k}}{\partial r}+\frac{(\Delta r)^{2}}{4 r_{i}^{2}} \frac{\partial^{2} U_{i, j, k}}{\partial r^{2}}-\frac{1}{2} \frac{(\Delta r)^{2}}{r_{i}^{4}} \frac{\partial^{2} U_{i, j, k}}{\partial \theta^{2}}+\frac{(\Delta r)^{2}}{4 r_{i}^{3}} \frac{\partial}{\partial r}\left(\frac{\partial^{2} U_{i, j, k}}{\partial \theta^{2}}\right) \\
& +\frac{(\Delta r)^{2}}{4 r_{i}} \frac{\partial}{\partial r}\left(\frac{\partial^{2} U_{i, j, k}}{\partial z^{2}}\right)
\end{aligned}
$$

Using (12), (13), and multiplying both sides of (11) by $12(\Delta r)^{2}$ and rearranging and simplifying further, we get

$$
\begin{aligned}
& (\Delta r)^{2}\left(24+\delta_{r}^{2}+\delta_{\theta}^{2}+\delta_{z}^{2}+\frac{3 \Delta r}{2 r_{i}} \delta_{2 r}\right) f_{i, j, k} \\
& =a_{0}(i) U_{i, j, k}+a_{1}(i) U_{i+1, j, k}+a_{2}(i) U_{i-1, j, k} \\
& \quad+a_{3}(i)\left(U_{i, j+1, k}+U_{i, j-1, k}\right)+a_{4}(i)\left(U_{i, j, k+1}+U_{i, j, k-1}\right)+a_{5}(i)\left(U_{i+1, j+1, k}+U_{i+1, j-1, k}\right) \\
& \quad+a_{6}(i)\left(U_{i-1, j+1, k}+U_{i-1, j-1, k}\right)+a_{7}(i)\left(U_{i+1, j, k+1}+U_{i+1, j, k-1}\right)+a_{8}(i)\left(U_{i-1, j, k+1}+U_{i-1, j, k-1}\right) \\
& \quad+a_{9}(i)\left(U_{i, j+1, k+1}+U_{i, j-1, k+1}+U_{i, j+1, k-1}+U_{i, j-1, k-1}\right)
\end{aligned}
$$

where

$$
\begin{gathered}
a_{0}(i)=-40\left(1+\alpha+\frac{\omega}{r_{i}^{2}}\right)-6 \frac{(\Delta r)^{2}}{r_{i}^{2}}+12 \frac{\omega}{r_{i}^{2}} \frac{(\Delta r)^{2}}{r_{i}^{2}} \\
a_{1}(i)=20-2 \alpha-\frac{2 \omega}{r_{i}^{2}}+8 \frac{\Delta r}{r_{i}}-\frac{3}{2}\left(\frac{\Delta r}{r_{i}}\right)^{3}+3\left(\frac{\Delta r}{r_{i}}\right)^{2}-3 \frac{\omega}{r_{i}^{2}} \frac{\Delta r}{r_{i}}-3 \alpha \frac{\Delta r}{r_{i}} \\
a_{2}(i)=20-2 \alpha-\frac{2 \omega}{r_{i}^{2}}-8 \frac{\Delta r}{r_{i}}+\frac{3}{2}\left(\frac{\Delta r}{r_{i}}\right)^{3}+3\left(\frac{\Delta r}{r_{i}}\right)^{2}+3 \frac{\omega}{r_{i}^{2}} \frac{\Delta r}{r_{i}}+3 \alpha \frac{\Delta r}{r_{i}} \\
a_{3}(i)=-2 \alpha+12 \frac{\omega}{r_{i}^{2}}-2 \quad a_{4}(i)=20 \alpha-\frac{2 \omega}{r_{i}^{2}}-2 \\
a_{5}(i)=1+\frac{\omega}{r_{i}^{2}}+\frac{\Delta r}{r_{i}}+\frac{3}{2} \frac{\omega}{r_{i}^{2}} \frac{\Delta r}{r_{i}} \quad a_{6}(i)=1+\frac{\omega}{r_{i}^{2}}-\frac{\Delta r}{r_{i}}-\frac{3}{2} \frac{\omega}{r_{i}^{2}} \frac{\Delta r}{r_{i}} \\
a_{7}(i)=1+\alpha+\frac{\Delta r}{r_{i}}+\frac{3}{2} \alpha \frac{\Delta r}{r_{i}} \quad a_{8}(i)=1+\alpha-\frac{\Delta r}{r_{i}}-\frac{3}{2} \alpha \frac{\Delta r}{r_{i}} \quad a_{9}(i)=\alpha+\frac{\omega}{r_{i}^{2}}
\end{gathered}
$$

The system of equations in (14) is a linear sparse system, and thereby when solving we save both work and storage compared with a general system of equations. Such savings are basically true of finite difference methods: they yield sparse systems because each equation involves only few variables.

To solve equation (14), consider first in the $\theta$ direction, next in the $z$ direction and lastly in the $r$ direction, and thus (14) can be written in matrix form as 


$$
A U=\mathcal{B}
$$

where

$$
A=\left(\begin{array}{cccccccc}
R_{1} & S_{1} & & & & & & \\
T_{2} & R_{2} & S_{2} & & & & & \\
& T_{3} & R_{3} & S_{3} & & & & \\
& & & & \ddots & & & \\
& & & & & T_{M-1} & R_{M-1} & S_{M-1} \\
& & & & & & T_{M} & R_{M}
\end{array}\right)
$$

and it has $M$ blocks and each is of order $N P$.

$$
\begin{aligned}
& R_{i}=\left(\begin{array}{ccccccccccc}
R_{i}^{\prime} & R_{i}^{\prime \prime} & & & & & & \\
R_{i}^{\prime \prime} & R_{i}^{\prime} & R_{i}^{\prime \prime} & & & & & \\
& R_{i}^{\prime \prime} & R_{i}^{\prime} & R_{i}^{\prime \prime} & & & & \\
& & & & \ddots & & & \\
& & & & & R_{i}^{\prime \prime} & R_{i}^{\prime} & R_{i}^{\prime \prime} \\
& & & & & & R_{i}^{\prime \prime} & R_{i}^{\prime}
\end{array}\right), S_{i}=\left(\begin{array}{cccccccc}
S_{i}^{\prime} & S_{i}^{\prime \prime} & & & & & & \\
S_{i}^{\prime \prime} & S_{i}^{\prime} & S_{i}^{\prime \prime} & & & & & \\
& S_{i}^{\prime \prime} & S_{i}^{\prime} & S_{i}^{\prime \prime} & & & & \\
& & & & \ddots & & & \\
& & & & & S_{i}^{\prime \prime} & S_{i}^{\prime} & S_{i}^{\prime \prime} \\
& & & & & & S_{i}^{\prime \prime} & S_{i}^{\prime}
\end{array}\right) \\
& T_{i}=\left(\begin{array}{ccccccccc}
T_{i}^{\prime} & T_{i}^{\prime \prime} & & & & & & \\
T_{i}^{\prime \prime} & T_{i}^{\prime} & T_{i}^{\prime \prime} & & & & & \\
& T_{i}^{\prime \prime} & T_{i}^{\prime} & T_{i}^{\prime \prime} & & & & \\
& & & & \ddots & & & \\
& & & & & T_{i}^{\prime \prime} & T_{i}^{\prime} & T_{i}^{\prime \prime} \\
& & & & & & T_{i}^{\prime \prime} & T_{i}^{\prime}
\end{array}\right)
\end{aligned}
$$

$R_{i}, S_{i}$, and $T_{i}$ are of order $N P$.

\section{For the domain $D_{1}$}

$$
\begin{aligned}
& R_{i}^{\prime}=\left(\begin{array}{llllllll}
a_{0}(i) & a_{3}(i) & & & & & \\
a_{3}(i) & a_{0}(i) & a_{3}(i) & & & & & \\
& a_{3}(i) & a_{0}(i) & a_{3}(i) & & & & \\
& & & & \ddots & & & \\
& & & & & a_{3}(i) & a_{0}(i) & a_{3}(i) \\
& & & & & & a_{3}(i) & a_{0}(i)
\end{array}\right) \\
& R_{i}^{\prime \prime}=\left(\begin{array}{llllllll}
a_{4}(i) & a_{9}(i) & & & & & & \\
a_{9}(i) & a_{4}(i) & a_{9}(i) & & & & & \\
& a_{9}(i) & a_{4}(i) & a_{9}(i) & & & & \\
& & & & \ddots & & & \\
& & & & & a_{9}(i) & a_{4}(i) & a_{9}(i) \\
& & & & & & a_{9}(i) & a_{4}(i)
\end{array}\right) \\
& S_{i}^{\prime}=\left(\begin{array}{llllllll}
a_{1}(i) & a_{5}(i) & & & & & & \\
a_{5}(i) & a_{1}(i) & a_{5}(i) & & & & & \\
& a_{5}(i) & a_{1}(i) & a_{5}(i) & & & & \\
& & & & \ddots & & a_{1}(i) & a_{5}(i) \\
& & & & & a_{5}(i) & a_{5}(i) & a_{1}(i)
\end{array}\right)
\end{aligned}
$$




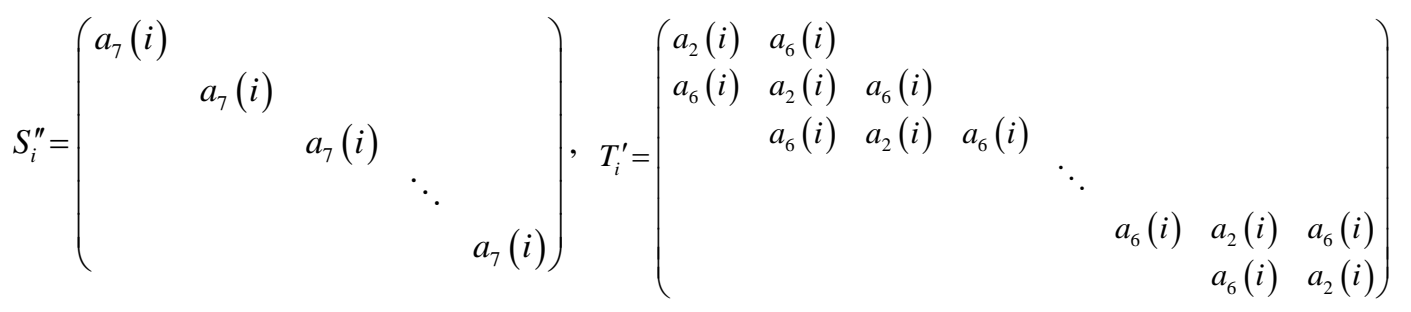

$$
\begin{aligned}
& T_{i}^{\prime \prime}=\left(\begin{array}{ccccc}
a_{8}(i) & & & & \\
& a_{8}(i) & & & \\
& & a_{8}(i) & & \\
& & & \ddots & \\
& & & & a_{8}(i)
\end{array}\right)
\end{aligned}
$$

\section{For the domain $D_{2}$,}

$$
\begin{aligned}
& R_{i}^{\prime}=\left(\begin{array}{llllllll}
a_{0}(i) & a_{3}(i) & & & & & & a_{3}(i) \\
a_{3}(i) & a_{0}(i) & a_{3}(i) & & & & & \\
& a_{3}(i) & a_{0}(i) & a_{3}(i) & & & \\
& & & & \ddots & & & \\
& & & & & a_{3}(i) & a_{0}(i) & a_{3}(i) \\
a_{3}(i) & & & & & & a_{3}(i) & a_{0}(i)
\end{array}\right) \\
& R_{i}^{\prime \prime}=\left(\begin{array}{llllllll}
a_{4}(i) & a_{9}(i) & & & & & & a_{9}(i) \\
a_{9}(i) & a_{4}(i) & a_{9}(i) & & & & & \\
& a_{9}(i) & a_{4}(i) & a_{9}(i) & & & & \\
& & & & \ddots & & & \\
& & & & & a_{9}(i) & a_{4}(i) & a_{9}(i) \\
a_{9}(i) & & & & & & a_{9}(i) & a_{4}(i)
\end{array}\right) \\
& S_{i}^{\prime}=\left(\begin{array}{llllllll}
a_{1}(i) & a_{5}(i) & & & & & & a_{5}(i) \\
a_{5}(i) & a_{1}(i) & a_{5}(i) & & & & & \\
& a_{5}(i) & a_{1}(i) & a_{5}(i) & & & & \\
& & & & \ddots & & & \\
& & & & & a_{5}(i) & a_{1}(i) & a_{5}(i) \\
a_{5}(i) & & & & & & a_{5}(i) & a_{1}(i)
\end{array}\right) \\
& T_{i}^{\prime}=\left(\begin{array}{llllllll}
a_{2}(i) & a_{6}(i) & & & & & & a_{6}(i) \\
a_{6}(i) & a_{2}(i) & a_{6}(i) & & & & & \\
& a_{6}(i) & a_{2}(i) & a_{6}(i) & & & \\
& & & & \ddots & & & \\
& & & & & a_{6}(i) & a_{2}(i) & a_{6}(i) \\
a_{6}(i) & & & & & & a_{6}(i) & a_{2}(i)
\end{array}\right)
\end{aligned}
$$

$S_{i}^{\prime \prime}$ and $T_{i}^{\prime \prime}$ are the same as in the domain $D_{1}$.

Here in $D_{2}$, the matrices $R_{i}^{\prime}, R_{i}^{\prime \prime}, S_{i}^{\prime}, S_{i}^{\prime \prime}, T_{i}^{\prime}$, and $T_{i}^{\prime \prime}$ are circulant matrices of order $N$; and

$$
\mathcal{B}=\left[\begin{array}{lllll}
\boldsymbol{B}_{0} & \boldsymbol{B}_{1} & \boldsymbol{B}_{2} & \cdots & \boldsymbol{B}_{M}
\end{array}\right]^{\mathrm{T}}, \quad \boldsymbol{B}_{i}=\left[\begin{array}{llllll}
\boldsymbol{d}_{i 1} & \boldsymbol{d}_{i 2} & \boldsymbol{d}_{i 3} & \cdots & \boldsymbol{d}_{i P}
\end{array}\right]^{\mathrm{T}} \text { and } \boldsymbol{d}_{i k}=\left[\begin{array}{llll}
d_{i j 1} & d_{i j 2} & \cdots & d_{i j \mathrm{P}}
\end{array}\right]^{\mathrm{T}}
$$

such that each $d_{i j k}$ represents a known boundary values of $U$ and values of $f$, and

$$
\boldsymbol{U}=\left[\begin{array}{lllll}
\boldsymbol{U}_{1} & \boldsymbol{U}_{2} & \boldsymbol{U}_{3} & \cdots & \boldsymbol{U}_{M}
\end{array}\right]^{\mathrm{T}}, \boldsymbol{U}_{i}=\left(\begin{array}{llllll}
U_{i 1} & U_{i 2} & U_{i 3} & \cdots & U_{i P}
\end{array}\right)^{\mathrm{T}} \text { and } U_{i j}=\left(\begin{array}{lllll}
U_{i j 1} & U_{i j 2} & U_{i j 3} & \cdots & U_{i j P}
\end{array}\right)^{\mathrm{T}}
$$


Thus, we write (15) as

$$
\begin{aligned}
& R_{1} \boldsymbol{U}_{1}+S_{1} \boldsymbol{U}_{2}=\boldsymbol{B}_{1} \\
& T_{2} \boldsymbol{U}_{1}+R_{2} \boldsymbol{U}_{2}+S_{2} \boldsymbol{U}_{3}=\boldsymbol{B}_{2} \\
& T_{3} \boldsymbol{U}_{2}+R_{3} \boldsymbol{U}_{3}+S_{3} \boldsymbol{U}_{4}=\boldsymbol{B}_{3} \\
& \cdots \\
& T_{M} \boldsymbol{U}_{M-1}+R_{M} \boldsymbol{U}_{M}=\boldsymbol{B}_{M}
\end{aligned}
$$

\section{Extended Hockney's Method}

Observe that matrices $R_{i}^{\prime}, R_{i}^{\prime \prime}, S_{i}^{\prime}$ and $T_{i}^{\prime}$ are real symmetric matrices and hence their eigenvalues and eigenvectors can easily be obtained as

For $D_{1}$

$$
\begin{gathered}
\lambda_{i j}=a_{0}(i)+2 a_{3}(i) \cos \left(\frac{j \pi}{N+1}\right), \quad \beta_{i j}=a_{4}(i)+2 a_{9}(i) \cos \left(\frac{j \pi}{N+1}\right), \quad \eta_{i j}=a_{1}(i)+2 a_{5}(i) \cos \left(\frac{j \pi}{N+1}\right) \\
\zeta_{i j}=a_{2}(i)+2 a_{6}(i) \cos \left(\frac{j \pi}{N+1}\right), \quad i=1(1) M \text { and } j=1(1) N
\end{gathered}
$$

and for $D_{2}$

$$
\begin{gathered}
\lambda_{i j}=a_{0}(i)+2 a_{3}(i) \cos \left(\frac{2 \pi j}{N}\right), \quad \beta_{i j}=a_{4}(i)+2 a_{9}(i) \cos \left(\frac{2 \pi j}{N}\right), \quad \eta_{i j}=a_{1}(i)+2 a_{5}(i) \cos \left(\frac{2 \pi j}{N}\right) \\
\zeta_{i j}=a_{2}(i)+2 a_{6}(i) \cos \left(\frac{2 \pi j}{N}\right), \quad i=1(1) M \text { and } j=1(1) N
\end{gathered}
$$

Let $\boldsymbol{q}_{j}$ be an eigenvector of $R_{i}^{\prime}, R_{i}^{\prime \prime}, S_{i}^{\prime}$ and $T_{i}^{\prime}$ corresponding to the eigenvalue $\lambda_{i j}, \beta_{i j}, \eta_{i j}$ and $\xi_{i j}$; and matrix $Q=\left[\begin{array}{lllll}\boldsymbol{q}_{1} & \boldsymbol{q}_{2} & \boldsymbol{q}_{3} & \cdots & \boldsymbol{q}_{N}\end{array}\right]^{\mathrm{T}}$ be a modal matrix of $R_{i}^{\prime}, R_{i}^{\prime \prime}, S_{i}^{\prime}$ and $T_{i}^{\prime}, \forall i$ such that $Q^{T} Q=I$

The $N \times N$ modal matrix $Q$ is defined by

$$
q_{i j}=\sqrt{\frac{2}{N+1}} \sin \left(\frac{i j \pi}{N+1}\right), i, j=1(1) N \text { for } D_{1} ; q_{i j}=\left(\frac{\cos \theta+\sin \theta}{\sqrt{N}}\right) \text { where } \theta=\frac{2 \pi}{N}(i-1)(j-1)
$$

$i, j=1(1) N$ for $D_{2}$

Let $\mathbb{Q}=\operatorname{diag}(Q, Q, Q, \cdots, Q)$ be a matrix of order $N P$; thus $\mathbb{Q}$ satisfy $\mathbb{Q}^{T} \mathbb{Q}=I$ since $Q^{T} Q=I$.

Since $R_{i}, S_{i}$ and $T_{i}$ are symmetric matrices, we have

$$
\begin{aligned}
& \mathbb{Q}^{T} R_{i} \mathbb{Q}=\operatorname{diag}\left(\mu_{j 1}^{i}, \mu_{j 2}^{i}, \cdots, \mu_{j P}^{i}\right)=\Upsilon_{i} \text { where } \mu_{j P}^{i}=\lambda_{i j}+2 \beta_{i j} \cos \left(\frac{k \pi}{P+1}\right) \\
& \mathbb{Q}^{T} S_{i} \mathbb{Q}=\operatorname{diag}\left(\xi_{j 1}^{i}, \xi_{j 2}^{i}, \cdots, \xi_{j P}^{i}\right)=\Phi_{i} \text { where } \mu_{j P}^{i}=\eta_{i j}+2 a_{7}(i) \cos \left(\frac{k \pi}{P+1}\right) \\
& \mathbb{Q}^{T} T_{i} \mathbb{Q}=\operatorname{diag}\left(\tau_{j 1}^{i}, \tau_{j 2}^{i}, \cdots, \tau_{j P}^{i}\right)=\Psi_{i} \text { where } \tau_{j P}^{i}=\zeta_{i j}+2 a_{8}(i) \cos \left(\frac{k \pi}{P+1}\right)
\end{aligned}
$$

Let

$$
\mathbb{Q}^{T} \boldsymbol{U}_{i}=\boldsymbol{V}_{i} \Rightarrow \boldsymbol{U}_{i}=\mathbb{Q} \boldsymbol{V}_{i}, \quad \mathbb{Q}^{T} \boldsymbol{B}_{i}=\overline{\boldsymbol{b}}_{i} \Rightarrow \boldsymbol{B}_{i}=\mathbb{Q} \overline{\boldsymbol{b}}_{i}
$$

where

$$
\begin{gathered}
\boldsymbol{V}_{i}=\left[\begin{array}{lllll}
V_{i 1} & V_{i 2} & V_{i 3} & \cdots & V_{i P}
\end{array}\right]^{\mathrm{T}}, V_{i k}=\left[\begin{array}{lllll}
v_{i 1 k} & v_{i 2 k} & v_{i 3 k} & \cdots & v_{i N k}
\end{array}\right]^{\mathrm{T}} ; \\
\overline{\boldsymbol{b}}_{i}=\left[\begin{array}{llll}
\boldsymbol{b}_{i 1} & \boldsymbol{b}_{i 2 ?} & \cdots & \boldsymbol{b}_{i k}
\end{array}\right]^{\mathrm{T}} \text { and } \boldsymbol{b}_{i k}=\left[\begin{array}{llll}
b_{i 1 k} & b_{i 2 k} & \cdots & b_{i N k}
\end{array}\right]^{\mathrm{T}}
\end{gathered}
$$


Pre-multiplying Equation (16) by $\mathbb{Q}^{T}$ and applying (17), we get

$$
\begin{aligned}
& \Upsilon_{1} \boldsymbol{V}_{1}+\Phi_{1} \boldsymbol{V}_{2}=\overline{\boldsymbol{b}}_{1} \\
& \Psi_{2} \boldsymbol{V}_{1}+\Upsilon_{2} \boldsymbol{V}_{2}+\Phi_{2} \boldsymbol{V}_{3}=\overline{\boldsymbol{b}}_{2} \\
& \Psi_{3} \boldsymbol{V}_{2}+\Upsilon_{3} \boldsymbol{V}_{3}+\Phi_{3} \boldsymbol{V}_{4}=\overline{\boldsymbol{b}}_{3} \\
& \ldots \\
& \Psi_{M} \boldsymbol{V}_{M-1}+\Upsilon_{M} \boldsymbol{V}_{M}=\overline{\boldsymbol{b}}_{M}
\end{aligned}
$$

Now from each Equation of (18) we collect the first equations and put them as one group of equation

$$
\begin{aligned}
& \mu_{j k}^{1} V_{k}^{1}+\xi_{j k}^{i} V_{k}^{2}=\overline{\boldsymbol{b}}_{1} \\
& \tau_{j k}^{2} V_{k}^{1}+\mu_{j k}^{2} V_{k}^{2}+\xi_{j k}^{2} V_{k}^{3}=\overline{\boldsymbol{b}}_{2} \\
& \tau_{j k}^{3} V_{k}^{2}+\mu_{j k}^{3} V_{k}^{3}+\xi_{j k}^{3} V_{k}^{4}=\overline{\boldsymbol{b}}_{3} \\
& \tau_{j k}^{M-1} V_{k}^{M-2}+\mu_{j k}^{M-1} V_{k}^{M-1}+\xi_{j k}^{M-1} V_{k}^{M}=\overline{\boldsymbol{b}}_{M-1} \\
& \tau_{j k}^{M} V_{k}^{M-1}+\mu_{j k}^{M} V_{k}^{M}=\overline{\boldsymbol{b}}_{M}
\end{aligned}
$$

Now put $k=1$ in Equation (19) and collect the entire first set of equations, for $i=1,2,3, \cdots, M$ and $j=1,2,3, \cdots, N$ to get

$$
\tau_{j 1}^{i} 1_{j 1}^{i-1}+\mu_{j 1}^{i} v_{j 1}^{i}+\xi_{j 1}^{i} v_{j 1}^{i+1}=\overline{\boldsymbol{b}}_{i} \text { and } v_{j 1}^{0}=0=v_{j 1}^{M}
$$

Again consider the second equations by putting $k=2$, and get

$$
\tau_{j 2}^{i} v_{j 2}^{i-1}+\mu_{j 2}^{i} v_{j 2}^{i}+\xi_{j 2}^{i} v_{j 2}^{i+1}=\overline{\boldsymbol{b}}_{i} \text { and } v_{j 2}^{0}=0=v_{j 2}^{M}
$$

Continuing in this manner and finally considering the last equations for $k=P$, we obtain

$$
\tau_{j P}^{i} v_{j P}^{i-1}+\mu_{j P}^{i} v_{j P}^{i}+\xi_{j P}^{i} v_{j P}^{i+1}=\overline{\boldsymbol{b}}_{i} \text { and } v_{j P}^{0}=0=v_{j P}^{M}
$$

All these set of Equations (20a)-(20c) are tri-diagonal ones and hence we solve for $v_{j k}^{i}$ by using Thomas algorithm. With the help of (17) again we get all $u_{j k}^{i}$ and this solves (14) as desired. By doing this we generally reduce the number of computations and computational time.

\section{Numerical Results}

In order to test the efficiency and adaptability of the proposed method, computational experiments are done on some selected problems that may arise in practice, for which the analytical solutions of $U$ are known to us. The computed solutions are found for all grid points for any values of $M, N$ and $P$. Here results are reported at some randomly taken mesh points in terms of the absolute maximum error from Table 1 to 7 .

Example 1. Consider $\nabla^{2} U=0$ with the boundary conditions $U(0, \theta, z)=0, U(1, \theta, z)=z \sin \theta$

$$
U(r, 0, z)=0=U(r, \pi, z) \text {, and } U(r, \theta, 0)=0, U(r, \theta, 1)=r \sin \theta
$$

The analytical solution is $U(r, \theta, z)=r z \sin \theta$ and the computed results of this example are shown in Table 1.

Example 2. Consider $\nabla^{2} U=-\pi^{2} r \cos \theta \sin \pi z$ with the boundary conditions

$$
\begin{gathered}
U(1, \theta, z)=\cos \theta \sin \pi z, U(2, \theta, z)=2 \cos \theta \sin \pi z \\
U(r, 0, z)=r \sin \pi z, U\left(r, \frac{\pi}{2}, z\right)=0, \text { and } U(r, \theta, 0)=0=U(r, \theta, 1)
\end{gathered}
$$

The analytical solution is $U(r, \theta, z)=r \cos \theta \sin \pi z$ and the computed results of this example are shown in Table 2.

Example 3. Consider $\nabla^{2} U=-3 \cos \theta$ with the boundary conditions

$$
U(0, \theta, z)=U(1, \theta, z)=-2 z, U(r, 0, z)=r(1-r)-2 z, U\left(r, \frac{\pi}{2}, z\right)=-2 z
$$


Table 1. Maximum absolute error of example 1.

\begin{tabular}{cccc}
\hline$(N, P, M)$ & Max. absolute error & $(N, P, M)$ & Max. absolute error \\
\hline$(9,9,9)$ & $3.51670 \mathrm{e}-005$ & $(29,9,39)$ & $1.37257 \mathrm{e}-006$ \\
$(9,9,29)$ & $1.46565 \mathrm{e}-005$ & $(29,19,9)$ & $4.15180 \mathrm{e}-006$ \\
$(9,19,9)$ & $3.53325 \mathrm{e}-005$ & $(29,29,19)$ & $2.45633 \mathrm{e}-006$ \\
$(9,19,19)$ & $2.06578 \mathrm{e}-005$ & $(29,29,29)$ & $1.74383 \mathrm{e}-006$ \\
$(9,29,39)$ & $1.13280 \mathrm{e}-005$ & $(29,39,19)$ & $2.45924 \mathrm{e}-006$ \\
$(9,39,29)$ & $1.46438 \mathrm{e}-005$ & $(29,39,29)$ & $1.74829 \mathrm{e}-006$ \\
$(19,9,9)$ & $9.21838 \mathrm{e}-006$ & $(39,9,19)$ & $1.35171 \mathrm{e}-006$ \\
$(19,9,19)$ & $5.32850 \mathrm{e}-006$ & $(39,9,39)$ & $7.75143 \mathrm{e}-007$ \\
$(19,19,19)$ & $5.46733 \mathrm{e}-006$ & $(39,19,29)$ & $9.82456 \mathrm{e}-007$ \\
$(19,29,39)$ & $3.02425 \mathrm{e}-006$ & $(39,29,19)$ & $1.38647 \mathrm{e}-006$ \\
$(19,39,9)$ & $9.27536 \mathrm{e}-006$ & $(39,39,9)$ & $2.34568 \mathrm{e}-006$ \\
$(19,39,39)$ & $3.02636 \mathrm{e}-006$ & $(39,39,39)$ & $7.68613 \mathrm{e}-007$ \\
\hline
\end{tabular}

Table 2. Maximum absolute error of example 2.

\begin{tabular}{cccc}
\hline$(N, P, M)$ & Max. absolute error & $(N, P, M)$ & Max. absolute error \\
\hline$(9,9,9)$ & $2.93159 \mathrm{e}-003$ & $(29,9,39)$ & $2.98714 \mathrm{e}-003$ \\
$(9,9,29)$ & $2.95649 \mathrm{e}-003$ & $(29,19,9)$ & $7.39877 \mathrm{e}-004$ \\
$(9,19,9)$ & $7.32025 \mathrm{e}-004$ & $(29,29,19)$ & $3.31950 \mathrm{e}-004$ \\
$(9,19,19)$ & $7.38648 \mathrm{e}-004$ & $(29,29,29)$ & $3.31771 \mathrm{e}-004$ \\
$(9,29,39)$ & $3.27574 \mathrm{e}-004$ & $(29,39,19)$ & $1.86718 \mathrm{e}-004$ \\
$(9,39,29)$ & $1.83450 \mathrm{e}-004$ & $(29,39,29)$ & $1.86618 \mathrm{e}-004$ \\
$(19,9,9)$ & $2.95328 \mathrm{e}-003$ & $(39,9,19)$ & $2.98618 \mathrm{e}-003$ \\
$(19,9,19)$ & $2.97861 \mathrm{e}-003$ & $(39,9,39)$ & $2.98710 \mathrm{e}-003$ \\
$(19,19,19)$ & $7.44907 \mathrm{e}-004$ & $(39,19,29)$ & $7.46353 \mathrm{e}-004$ \\
$(19,29,39)$ & $3.31145 \mathrm{e}-004$ & $(39,29,19)$ & $3.31953 \mathrm{e}-004$ \\
$(19,39,9)$ & $1.84585 \mathrm{e}-004$ & $(39,39,9)$ & $1.84916 \mathrm{e}-004$ \\
$(19,39,39)$ & $1.86232 \mathrm{e}-004$ & $(39,39,39)$ & $1.86784 \mathrm{e}-004$ \\
\hline
\end{tabular}

$$
U(r, \theta, 0)=r(1-r) \cos \theta, U(r, \theta, 1)=r(1-r) \cos \theta-2
$$

The analytical solution is $U(r, \theta, z)=r(1-r) \cos \theta-2 z$ and the computed results of this example are shown in Table 3.

Example 4. Consider $\nabla^{2} U=-\pi^{2}\left(r^{2}-\frac{1}{r^{2}}\right) \sin (2 \theta) \sin (\pi z)$ with the boundary conditions

$$
U(1, \theta, z)=0, U(2, \theta, z)=\frac{15}{4} \sin (2 \theta) \sin (\pi z), U(r, 0, z)=0=U\left(r, \frac{\pi}{2}, z\right) \text { and } U(r, \theta, 0)=0=U(r, \theta, 1)
$$

The analytical solution is $U(r, \theta, z)=\left(r^{2}-\frac{1}{r^{2}}\right) \sin (2 \theta) \sin (\pi z)$ and the computed results of this example are shown in Table 4.

Example 5 Consider $\nabla^{2} U=\left(8 r z(1-z)-2 r^{3}\right)(\sin \theta+\cos \theta)$, where $0 \leq \theta<2 \pi$ with the boundary conditions

$$
U(0, \theta, z)=0, U(1, \theta, z)=z(1-z)(\sin \theta+\cos \theta) \quad U(r, \theta, 0)=0=U(r, \theta, 1)
$$

The analytical solution is $U(r, \theta, z)=r^{3} z(1-z)(\sin \theta+\cos \theta)$ and the computed results of this example are shown in Table 5. 
Table 3. Maximum absolute error of example 3.

\begin{tabular}{cccc}
\hline$(N, P, M)$ & Max. absolute error & $(N, P, M)$ & Max. absolute error \\
\hline$(9,9,9)$ & $1.81124 \mathrm{e}-004$ & $(29,9,39)$ & $1.16544 \mathrm{e}-005$ \\
$(9,9,29)$ & $4.45263 \mathrm{e}-005$ & $(29,19,9)$ & $1.82484 \mathrm{e}-004$ \\
$(9,19,9)$ & $1.81185 \mathrm{e}-004$ & $(29,29,19)$ & $4.61297 \mathrm{e}-005$ \\
$(9,19,19)$ & $6.02480 \mathrm{e}-005$ & $(29,29,29)$ & $2.04978 \mathrm{e}-005$ \\
$(9,29,39)$ & $3.97430 \mathrm{e}-005$ & $(29,39,19)$ & $4.61300 \mathrm{e}-005$ \\
$(9,39,29)$ & $4.46327 \mathrm{e}-005$ & $(29,39,29)$ & $2.04979 \mathrm{e}-005$ \\
$(19,9,9)$ & $1.81939 \mathrm{e}-004$ & $(39,9,19)$ & $4.61828 \mathrm{e}-005$ \\
$(19,9,19)$ & $4.59426 \mathrm{e}-005$ & $(39,9,39)$ & $1.17058 \mathrm{e}-005$ \\
$(19,19,19)$ & $4.59583 \mathrm{e}-005$ & $(39,19,29)$ & $2.05467 \mathrm{e}-005$ \\
$(19,29,39)$ & $1.50833 \mathrm{e}-005$ & $(39,29,19)$ & $4.61879 \mathrm{e}-005$ \\
$(19,39,9)$ & $1.82013 \mathrm{e}-004$ & $(39,39,9)$ & $1.82652 \mathrm{e}-004$ \\
$(19,39,39)$ & $1.50852 \mathrm{e}-005$ & $(39,39,39)$ & $1.15493 \mathrm{e}-005$ \\
\hline
\end{tabular}

Table 4. Maximum absolute error of example 4.

\begin{tabular}{cccc}
\hline$(N, P, M)$ & Max. absolute error & $(N, P, M)$ & Max. absolute error \\
\hline$(9,9,9)$ & $3.68396 \mathrm{e}-003$ & $(29,9,39)$ & $3.98135 \mathrm{e}-003$ \\
$(9,9,29)$ & $4.07400 \mathrm{e}-003$ & $(29,19,9)$ & $6.33780 \mathrm{e}-004$ \\
$(9,19,9)$ & $7.68229 \mathrm{e}-004$ & $(29,29,19)$ & $3.64070 \mathrm{e}-004$ \\
$(9,19,19)$ & $1.04366 \mathrm{e}-003$ & $(29,29,29)$ & $4.17368 \mathrm{e}-004$ \\
$(9,29,39)$ & $5.73867 \mathrm{e}-004$ & $(29,39,19)$ & $1.75928 \mathrm{e}-004$ \\
$(9,39,29)$ & $3.62888 \mathrm{e}-004$ & $(29,39,29)$ & $2.24720 \mathrm{e}-004$ \\
$(19,9,9)$ & $3.58663 \mathrm{e}-003$ & $(39,9,19)$ & $3.89251 \mathrm{e}-003$ \\
$(19,9,19)$ & $3.92179 \mathrm{e}-003$ & $(39,9,39)$ & $3.97355 \mathrm{e}-003$ \\
$(19,19,19)$ & $9.34774 \mathrm{e}-004$ & $(39,19,29)$ & $9.60868 \mathrm{e}-004$ \\
$(19,29,39)$ & $4.61633 \mathrm{e}-004$ & $(39,29,19)$ & $3.55183 \mathrm{e}-004$ \\
$(19,39,9)$ & $7.29565 \mathrm{e}-004$ & $(39,39,9)$ & $7.23913 \mathrm{e}-004$ \\
$(19,39,39)$ & $2.68695 \mathrm{e}-004$ & $(39,39,39)$ & $2.34933 \mathrm{e}-004$ \\
\hline
\end{tabular}

Table 5. Maximum absolute error of example 5.

\begin{tabular}{cccc}
\hline$(N, P, M)$ & Max. absolute error & $(N, P, M)$ & Max. absolute error \\
\hline$(9,9,9)$ & $5.97062 \mathrm{e}-004$ & $(29,9,39)$ & $1.65910 \mathrm{e}-004$ \\
$(9,9,29)$ & $4.42157 \mathrm{e}-004$ & $(29,19,9)$ & $4.11093 \mathrm{e}-004$ \\
$(9,19,9)$ & $5.09956 \mathrm{e}-004$ & $(29,29,19)$ & $1.01380 \mathrm{e}-004$ \\
$(9,19,19)$ & $3.72361 \mathrm{e}-004$ & $(29,29,29)$ & $6.92680 \mathrm{e}-005$ \\
$(9,29,39)$ & $3.26827 \mathrm{e}-004$ & $(29,39,19)$ & $1.03392 \mathrm{e}-004$ \\
$(9,39,29)$ & $3.27891 \mathrm{e}-004$ & $(29,39,29)$ & $6.38312 \mathrm{e}-005$ \\
$(19,9,9)$ & $3.72181 \mathrm{e}-004$ & $(39,9,19)$ & $1.80739 \mathrm{e}-004$ \\
$(19,9,19)$ & $2.39220 \mathrm{e}-004$ & $(39,9,39)$ & $1.49613 \mathrm{e}-004$ \\
$(19,19,19)$ & $1.52973 \mathrm{e}-004$ & $(39,19,29)$ & $6.95506 \mathrm{e}-005$ \\
$(19,29,39)$ & $1.04227 \mathrm{e}-004$ & $(39,29,19)$ & $1.06985 \mathrm{e}-004$ \\
$(19,39,9)$ & $3.96923 \mathrm{e}-004$ & $(39,39,9)$ & $4.28673 \mathrm{e}-004$ \\
$(19,39,39)$ & $9.84850 \mathrm{e}-005$ & $(39,39,39)$ & $3.91182 \mathrm{e}-005$ \\
\hline
\end{tabular}


This example was considered by M.C. Lai [1] as a test problem and our results are better than their results in terms of accuracy. For instance, for $(8,16,16)$ the maximum absolute error in their result is 9.1438e-004 and while ours is 3.28689e-004.

Example 6 Consider $\nabla^{2} U=6 r z \cos \theta$, where $0 \leq \theta<2 \pi$ with the boundary conditions

$$
U(0, \theta, z)=0, U(1, \theta, z)=z \cos ^{3} \theta ; U(r, \theta, 0)=0 \text { and } U(r, \theta, 1)=r^{3} \cos ^{3} \theta
$$

The analytical solution is $U(r, \theta, z)=r^{3} z \cos ^{3} \theta$ and the computed results are shown in Table 6.

Example 5.7 Consider $\nabla^{2} U=-\pi^{2}\left(r^{2}-\frac{1}{r^{2}}\right) \sin (2 \theta) \sin (\pi z)$ where $0 \leq \theta<2 \pi$ with the boundary conditions

$$
U(1, \theta, z)=0, \quad U(2, \theta, z)=\frac{15}{4} \sin (2 \theta) \sin (\pi z) ; U(r, \theta, 0)=0=U(r, \theta, 1)
$$

The analytical solution is $U(r, \theta, z)=\left(r^{2}-\frac{1}{r^{2}}\right) \sin (2 \theta) \sin (\pi z)$ and the computed results of this example are shown in Table 7.

Table 6. Maximum absolute error of example 6.

\begin{tabular}{cccc}
\hline$(N, P, M)$ & Max. absolute error & $(N, P, M)$ & Max. absolute error \\
\hline$(9,9,9)$ & $3.04648 \mathrm{e}-003$ & $(29,9,39)$ & $3.06543 \mathrm{e}-004$ \\
$(9,9,29)$ & $3.18297 \mathrm{e}-003$ & $(29,19,9)$ & $2.00777 \mathrm{e}-004$ \\
$(9,19,9)$ & $3.05549 \mathrm{e}-003$ & $(29,29,19)$ & $2.85659 \mathrm{e}-004$ \\
$(9,19,19)$ & $3.16606 \mathrm{e}-003$ & $(29,29,29)$ & $3.02059 \mathrm{e}-004$ \\
$(9,29,39)$ & $3.19893 \mathrm{e}-003$ & $(29,39,19)$ & $2.85718 \mathrm{e}-004$ \\
$(9,39,29)$ & $3.19459 \mathrm{e}-003$ & $(29,39,29)$ & $3.02122 \mathrm{e}-004$ \\
$(19,9,9)$ & $6.03143 \mathrm{e}-004$ & $(39,9,19)$ & $1.42033 \mathrm{e}-004$ \\
$(19,9,19)$ & $6.87721 \mathrm{e}-004$ & $(39,9,39)$ & $1.62951 \mathrm{e}-004$ \\
$(19,19,19)$ & $6.89766 \mathrm{e}-004$ & $(39,19,29)$ & $1.58004 \mathrm{e}-004$ \\
$(19,29,39)$ & $7.13568 \mathrm{e}-004$ & $(39,29,19)$ & $1.42553 \mathrm{e}-004$ \\
$(19,39,9)$ & $6.05428 \mathrm{e}-004$ & $(39,39,9)$ & $1.58596 \mathrm{e}-004$ \\
$(19,39,39)$ & $7.13712 \mathrm{e}-004$ & $(39,39,39)$ & $1.63583 \mathrm{e}-004$ \\
\hline
\end{tabular}

Table 7. Maximum absolute error of example 7.

\begin{tabular}{cccc}
\hline$(N, P, M)$ & Max. absolute error & $(N, P, M)$ & Max. absolute error \\
\hline$(9,9,9)$ & $3.00418 \mathrm{e}-003$ & $(29,9,39)$ & $4.13706 \mathrm{e}-003$ \\
$(9,9,29)$ & $2.36262 \mathrm{e}-003$ & $(29,19,9)$ & $8.41886 \mathrm{e}-004$ \\
$(9,19,9)$ & $4.54924 \mathrm{e}-003$ & $(29,29,19)$ & $5.78646 \mathrm{e}-004$ \\
$(9,19,19)$ & $4.13088 \mathrm{e}-003$ & $(29,29,29)$ & $6.30456 \mathrm{e}-004$ \\
$(9,29,39)$ & $4.49099 \mathrm{e}-003$ & $(29,39,19)$ & $3.91287 \mathrm{e}-004$ \\
$(9,39,29)$ & $4.67590 \mathrm{e}-003$ & $(29,39,29)$ & $4.41870 \mathrm{e}-004$ \\
$(19,9,9)$ & $3.54731 \mathrm{e}-003$ & $(39,9,19)$ & $4.01710 \mathrm{e}-003$ \\
$(19,9,19)$ & $3.84938 \mathrm{e}-003$ & $(39,9,39)$ & $4.09806 \mathrm{e}-003$ \\
$(19,19,19)$ & $1.08325 \mathrm{e}-003$ & $(39,19,29)$ & $1.10354 \mathrm{e}-003$ \\
$(19,29,39)$ & $6.43373 \mathrm{e}-004$ & $(39,29,19)$ & $5.01257 \mathrm{e}-004$ \\
$(19,39,9)$ & $9.83307 \mathrm{e}-004$ & $(39,39,9)$ & $7.61254 \mathrm{e}-004$ \\
$(19,39,39)$ & $4.66590 \mathrm{e}-004$ & $(39,39,39)$ & $3.82100 \mathrm{e}-004$ \\
\hline
\end{tabular}




\section{Conclusions}

In this work, we have transformed the three dimensional Poisson's equation in cylindrical coordinates system into a system of algebraic linear equations using its equivalent fourth-order finite difference approximation scheme. The resulting large number of algebraic equation is, then, systematically arranged in order to get a block matrix. By extending Hockney's method to three dimensions, we reduced the obtained matrix into a block tridiagonal matrix, and each block is solved by the help of Thomas algorithm. We have successfully implemented this method to find the solution of the three dimensional Poisson's equation in cylindrical coordinates system and it is found that the method can easily be applied and adapted to find a solution of some related applied problems. The method produced accurate results considering double precision. This method is direct and allows considerable savings in computer storage as well as execution speed.

Therefore, the method is suitable to apply to some three dimensional Poisson's equations.

\section{References}

[1] Lai, M.C. (2002) A Simple Compact Fourth-Order Poisson Solver on Polar Geometry. Journal of Computational Physics, 182, 337-345. http://dx.doi.org/10.1006/jcph.2002.7172

[2] Mittal, R.C and Gahlaut, S. (1987) High Order Finite Difference Schemes to Solve Poisson's Equation in Cylindrical Symmetry. Communications in Applied Numerical Methods, 3, 457-461.

[3] Mittal, R.C. and Gahlaut, S. (1991) High-Order Finite Differences Schemes to Solve Poisson's Equation in Polar Coordinates. IMA Journal of Numerical Analysis, 11, 261-270. http://dx.doi.org/10.1093/imanum/11.2.261

[4] Alemayehu, S. and Mittal, R.C. (2013) Fast Finite Difference Solutions of the Three Dimensional Poisson's Equation in Cylindrical Coordinates. American Journal of Computational Mathematics, 3, 356-361.

[5] Tan, C.S. (1985) Accurate Solution of Three Dimensional Poisson's Equation in Cylindrical Coordinate by Expansion in Chebyshev Polynomials. Journal of Computational Physics, 59, 81-95. http://dx.doi.org/10.1016/0021-9991(85)90108-1

[6] Iyengar, S.R.K. and Manohar, R. (1988) High Order Difference Methods for Heat Equation in Polar Cylindrical Polar Cylindrical Coordinates. Journal of Computational Physics, 77, 425-438. http://dx.doi.org/10.1016/0021-9991(88)90176-3

[7] Iyengar, S.R.K. and Goyal, A. (1990) A Note on Multigrid for the Three-Dimensional Poisson Equation in Cylindrical Coordinates. Journal of Computational and Applied Mathematics, 33, 163-169. http://dx.doi.org/10.1016/0377-0427(90)90366-8

[8] Lai, M.C. and Tseng, J.M. (2007) A formally Fourth-Order Accurate Compact Scheme for 3D Poisson Equation in Cylindrical and Spherical Coordinates. Journal of Computational and Applied Mathematics, 201, 175-181. http://dx.doi.org/10.1016/j.cam.2006.02.011

[9] Smith, G.D. (1985) Numerical Solutions of Partial Differential Equations: Finite Difference Methods. Third Edition. Oxford University Press, New York.

[10] Malcolm, M.A. and Palmer, J. (1974) A Fast Method for Solving a Class of Tri-Diagonal Linear Systems. Communications of Association for Computing Machinery, 17, 14-17. http://dx.doi.org/10.1145/360767.360777

[11] Hockney, R.W. (1965) A Fast Direct Solution of Poisson Equation Using Fourier Analysis. Journal of Alternative and Complementary Medicine, 12, 95-113. http://dx.doi.org/10.1145/321250.321259 